\title{
A genetic risk factor for thrombophilia in a Han Chinese family
}

\author{
GUOPING SUN ${ }^{1 *}$, YICONG JIA ${ }^{2 *}$, JINGYE MENG $^{3}$, MINGLIN OU $^{4}$, PENG ZHU ${ }^{1}$, \\ SHAN CONG ${ }^{4}$, YADAN LUO ${ }^{4}$, WEIGUO SUI ${ }^{4}$ and YONG DAI $^{5}$
}

\begin{abstract}
${ }^{1}$ Central Laboratory, Shenzhen Pingshan People's Hospital, Shenzhen, Guangdong 518118; ${ }^{2}$ Department of Genetics,
The Life Science College, Guangxi Normal University, Guilin, Guangxi 541002; ${ }^{3}$ Department of Hematology,

The Third People's Hospital of Shenzhen, Shenzhen, Guangdong 518020; ${ }^{4}$ Department of Nephrology, Guilin 181st Hospital,

Guangxi Key Laboratory of Metabolic Diseases Research, Guilin, Guangxi 541002; ${ }^{5}$ Clinical Medical Research Center,

The Second Clinical Medical College, Jinan University, Shenzhen People's Hospital, Shenzhen, Guangdong 518020, P.R. China
\end{abstract}

Received December 18, 2015; Accepted December 12, 2016

DOI: $10.3892 / \mathrm{mmr} .2017 .6217$

\begin{abstract}
Thrombophilia is a multifactorial disorder involving environmental and genetic factors. Well-known factors that result in predisposition to congenital disorders associated with thrombophilia include antithrombin deficiency, protein C and $\mathrm{S}$ deficiency, Factor $\mathrm{V}$ Leiden mutation, abnormal prothrombin and antiphospholipid syndrome. The present study revealed an association between a mutation of the $F 2$ gene, which codes for coagulation factor II, thrombin, and the risk of thrombophilia in a Han Chinese family, of which four members (I-2, II-2, II-3 and III-1) had a history of deep venous thromboembolism. The disease was measured in this family using laboratory measurements and computed tomography angiography. To identify the abnormality underlying the increased thrombophilia risk, whole-exome sequencing technology was used to analyze two affected individuals (II-2 and III-1). An exonic missense F2 mutation, T165M (NM_000506:c.C494T:p.T165M;rs5896), was identified from a total of 2,222 and 2,203 genetic variations observed in the two affected individuals, respectively, which were subsequently filtered and confirmed using Sanger sequencing. I-2, II-3 and III-1 shared this mutation with the proband (II-2), and II-6 had a heterozygous form of the mutation. This
\end{abstract}

Correspondence to: Professor Weiguo Sui, Department of Nephrology, Guilin 181st Hospital, Guangxi Key Laboratory of Metabolic Diseases Research, 1 Xinqiao Road, Guilin, Guangxi 541002, P.R. China

E-mail: suiwg@163.com

Professor Yong Dai, Clinical Medical Research Center, The Second Clinical Medical College, Jinan University, Shenzhen People's Hospital, 1017 Dongmen North Road, Shenzhen, Guangdong 518020, P.R. China

E-mail: daiyong22@aliyun.com

*Contributed equally

Key words: thrombophilia, Han Chinese, CT angiography, F2 gene deleterious mutation was not identified in normal controls. The present study may improve understanding of the function of the $F 2$ gene.

\section{Introduction}

Thrombophilia is a blood coagulation disorder characterized by an increased tendency to form clots, and results in a predisposition to adverse thrombotic events, including deep venous thromboembolism (DVT) and pulmonary embolism (PE). The risk factors for thrombophilia are genetic and environmental (1). Previous research has indicated that thrombophilia is the result of a deficiency in protein differentiation or function, including protein $\mathrm{C}$ and $\mathrm{S}$, and mutations in $\sim 8$ human genes have been identified as causative (2-4). However, the genetic basis of thrombophilia requires further investigation (1). A 52-year-old Han Chinese man was referred to the Shenzhen People's Hospital (Shenzen, China), due to recurrent DVT, with the first hospitalization for DVT occurring in 2001. Following treatment, the patient recovered. In 2003, the patient was readmitted due to $\mathrm{PE}$ and an inferior vena cava filter was placed in order to prevent further PE in Shenzhen People's Hospital. The patient's illness was diagnosed as thrombophilia following laboratory measurements and computed tomography (CT) angiography. The factors that may be involved in his disease were analyzed. In order to investigate the possible underlying causes, whole-exome sequencing (WES) technology was used to research the genetic factors, and a mutation in the $F 2$ gene was identified. The results of mutation detection in the Tianjin Institute of Hematology (Tianjin, China) validated this finding. No Factor V Leiden or prothrombin G20210A mutations were detected.

The $F 2$ gene has been assigned to the chromosomal region 11p11-q12 and encompasses $20.3 \mathrm{~kb}$ (5). This gene is transcribed and translated to produce coagulation factor II, thrombin (462 amino acids), which is involved in the final stage of blood coagulation in humans and controls synthesis of prothrombin $(5,6)$. F 2 mutations lead to various forms of thrombosis and dysprothrombinemia. However, in Asians, including Chinese populations, F2 mutations are rare. Little was known regarding the genetic background of DVT, however, previous research has since revealed common genetic risk factors in 
DVT (7-9). This research did not focus on Chinese populations, and the underlying molecular mechanisms remain to be elucidated. This evidence suggests that the study of genetic risk factors in this Chinese family is vital for the further understanding of thrombophilia. WES technology was used to examine two affected individuals from the same family as the proband to study the genetic risk factor for thrombophilia. The results of the present study may explain the underlying molecular mechanisms of thrombophilia in this family.

\section{Patients and methods}

Human samples. In order to investigate the association between the familial disease and its molecular mechanisms, a Han Chinese family with thrombophilia was recruited. The family comprised of 11 living members (including the proband and the affected individuals II-3, III-1) and 100 normal Han Chinese donors were also examined. The age range (35 to 60) and gender (50 males, 50 females) of normal donors matched the selection of patients II-2, II-3, III-1 with the disease and their respective ages were 52,50 and 32 . The diagnosis of thrombophilia was based on typical clinical and laboratory measurements and ascertained by CT angiography (10). Peripheral blood was collected in anticoagulation tubes from all study participants and genomic DNA was extracted from leukocytes of family members and normal donors using the phenol-chloroform protocol, following standard procedures (11). The protocol of the present study was approved by the Ethics Committee of The Second Clinical Medical College, Jinan University, Shenzhen People's Hospital (Shenzen, China) and written informed consent was obtained from all the participants.

Whole exome sequencing. The DNA of the proband and his son (III-1) was sequenced using the WES technique. The exons of $\sim 26,000$ protein coding genes were sequenced in matched thrombophilia and DNA of unaffected individuals. The coding sequences from each sample were captured using the NimbleGen SeqCap EZ Human Exome Library v2.0 kit (Roche Diagnostics, Indianapolis, IN, USA), and each captured library was loaded on to a Solexa Hiseq2000 platform (Illumina, Inc., San Diego, CA, USA) for sequence analysis. Genomic DNA samples from the two affected individuals were fragmented with the use of Covaris S2 system (Covaris Inc., Woburn, Massachusetts, USA). The adapters from DNA Library Prep Reagent Set (New England Biolabs Inc., USA) then were ligated to each end of the resulting fragments. The captured fragments were amplified by ligation-mediated polymerase chain reaction (LM-PCR) and hybridized to the Nimblegen SeqCap EZ Library so that enriched, non-hybridized fragments were washed out (12). Capture efficiency was evaluated by quantitative PCR (qPCR) using SYBR green setting on the real-time instrument (Roche Light Cycler 480, Indianapolis, IN, USA) (13). The primers of LM-PCR and qPCR were obtained from Multiplex Oligos Kit (New England Biolabs Inc., Ipswich, MA, USA) and NEBNext Library Quant Kit (New England Biolabs Inc.), respectively. The conditions of LM-PCR and qPCR were provided in the Tables I and II. Each captured library was then loaded on the Illumina Genome Analyzer (Illumina, Inc.) to ensure that the 2 samples met the desired average sequencing depth of $84 x$. Sequencing reads were analyzed by using the Solexa Hiseq2000 platform (Illumina, Inc.).

Read mapping and variant analysis. WES of the participants was conducted from genomic DNA isolated from blood and was submitted to the Huada Gene Research Institute (Shenzen, China) for analysis. Reads were aligned using the Burrows-Wheeler Aligner to the human reference genome (build 37) (14). Alignments in the combined BAM file were tested for single nucleotide polymorphisms (SNPs) and insertions or deletions (indels) using the SOAPsnp package and SOAP denovo respectively (www.soap.genomics.org. cn/soapsnp.html).

Candidate SNPs were filtered and flagged using the following modifications: i) SNP quality is $>20$; ii) the sequencing depth is not $<4 \mathrm{x}$; iii) the distance between two SNPs is $>5 \mathrm{bp}$; and iv) the estimated copy number is $<2$.

Candidate gene validation. The filtering of candidate mutations requires further validation. The reliability of WES was verified by Sanger sequencing to confirm the mutations of candidate genes. The PCR primers used were, forward, 5'-TGTGAACATCACCCGGTCAG-3' and reverse, 5'-GAC TGACGCCAGCTCTGAAG-3', and were designed to validate the candidate mutation in the gene of T165M. The results were consistent with the candidate mutations identified by WES. The reliability of WES results were also verified by these methods: i) Within-family validation, the co-segregated mutations of phenotype and genotype were observed in the genomes of each family member; and ii) Validation in 100 normal donors, validation that the co-segregated mutations of phenotype and genotype were not observed in normal donors. Furthermore, the cross-species amino acid comparison of coagulation factor II was analyzed by ClustalW2 (http://www.ebi. ac.uk/Tools/msa/clustalw2/). The amino acid sequences of F2 were obtained from the NCBI database, and then entered into ClustalW2. The result of ClustalW2 revealed the most appropriate match for the selected sequences and aligned them in such a way that the identities, similarities and differences were easily identified and understood.

\section{Results}

Clinical characteristics of the studied family with thrombophilia. The genetic factors that increase the risk of a thrombotic event are well known, and include deficiencies of protein $\mathrm{C}$ and protein $\mathrm{S}$. Factor $\mathrm{V}$ Leiden and prothrombin G20210A mutations, elevated levels of factors VIII, IX or XI, homocysteine, and fibrinogen may also be risk factors for a thrombotic event. Thrombophilia was confirmed by examining laboratory measurements and CT angiography in Shenzhen People's Hospital. The levels of factors VIII, IX and XI were measured by using enzyme-linked immunosorbent assays, a one-stage coagulation assay and a monoclonal antifactor XI capture antibody, respectively. Protein $\mathrm{C}$ and protein $\mathrm{S}$ were measured by a polyclonal enzyme-linked immunosorbent assay and the coagulation study was measured by the STAGO automatic coagulometer. Blood coagulation factor and protein $\mathrm{C}$ and $\mathrm{S}$ levels were normal. However, fibrinogen 
Table I. Thermocycling conditions of ligation mediated polymerase chain reaction.

\begin{tabular}{lccc}
\hline Cycle step & Temperature & Time & Cycles \\
\hline Initial denaturation & $98^{\circ} \mathrm{C}$ & $30 \mathrm{sec}$ & 1 \\
Denaturation & $98^{\circ} \mathrm{C}$ & $10 \mathrm{sec}$ & \\
Annealing/extension & $65^{\circ} \mathrm{C}$ & $75 \mathrm{sec}$ & 4 \\
Final extension & $65^{\circ} \mathrm{C}$ & $5 \mathrm{~min}$ & 1 \\
Hold & $4^{\circ} \mathrm{C}$ & $\infty$ & \\
\hline
\end{tabular}

Table II. Thermocycling conditions of quantitative polymerase chain reaction.

\begin{tabular}{lccc}
\hline Cycle step & Temperature & Time & Cycles \\
\hline Initial denaturation & $95^{\circ} \mathrm{C}$ & $1 \mathrm{~min}$ & 1 \\
Denaturation & $95^{\circ} \mathrm{C}$ & $15 \mathrm{sec}$ & \\
Extension & $63^{\circ} \mathrm{C}$ & $45 \mathrm{sec}$ & 35 \\
\hline
\end{tabular}

levels were abnormal in the proband and other affected family members. Their activated partial thromboplastin time and plasma prothrombin time were observed to be prolonged. Plasma immunoreactive fibrinogen levels were also lower (Table III).

The proband underwent combined CT angiography and CT venography of the stomach and splenomegaly and cirrhosis was observed (Figs. 1 and 2). CT is a form of imaging that is used as part of the daily clinical routine to detect DVT and PE.

Whole exome sequencing identified F2 as a candidate gene. The exomes of the proband and his son (II-2 and III-1) were captured and sequenced. Following the sequencing and filtering as described previously, candidate variants were identified for the two affected individuals. Overall, high coverage of the exome was attained, 93 and 99 million sequencing reads were produced for two affected individuals, comprising 7.3 and 7.4 billion bases, respectively. In total, $\sim 90.7 \%$ of these were aligned to the human reference genome and $60.5 \%$ of these fell onto targeted and enriched exons. The average sequence read depth was $84 \mathrm{x}$ in targeted exons. A total of 101,037 and 97,227 genetic variations, including nonsynonymous mutations, splice-acceptor and splice-donor site variations, and indels were identified in the two affected individuals in the family, respectively. Furthermore, these variants of II-2 and III-1 were further evaluated by National Center for Biotechnology Information (NCBI) dbSNP build 132 (http://www.ncbi.nlm.nih.gov/projects/SNP/), SIFT (http://blocks.fhcrc.org/sift/SIFT.html.) and PolyPhen2 (http://genetics.bwh.harvard.edu/pph2/). SIFT and PolyPhen2 programs were used for protein analysis. The applied process of SIFT and PolyPhen2 were compliant with the previously described protocols $(15,16)$. Following this filtration, only one variant (NM_000506:c.C494T:p.T165M;rs5896) was located in the candidate region. The variant is a homozygote missense mutation that has not previously been observed in Han Chinese

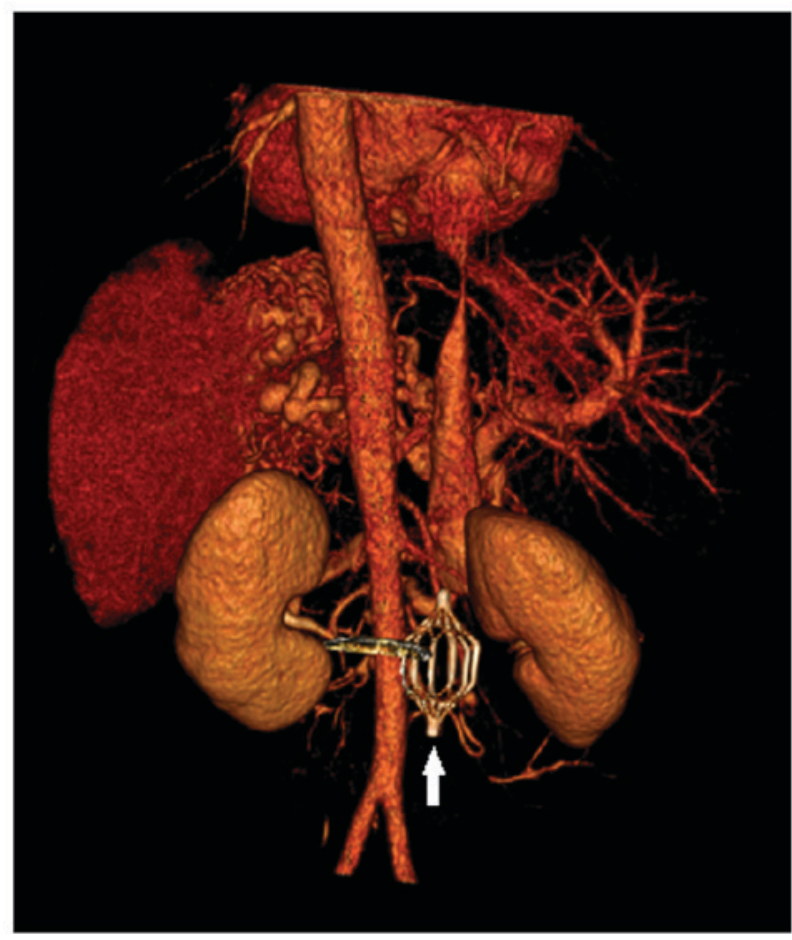

Figure 1. Computed tomography angiography image of the proband. The arrow indicates the inferior vena cava filter.

population by other researchers, but this variant was reported as a genetic risk factor associated with thrombophilia in the Xinjiang Kazakh population (17).

Sanger sequencing. To validate the findings of WES, Sanger sequencing was used to analyze the candidate mutation (T165 M) in F2 in the family. As a result, the Sanger sequencing further confirmed the candidate variant (T165M) did not result in the possibility of a false positive. To further study the characterization of the variant, Sanger sequencing was used to analyze the mutation in $F 2$ in other family members and 100 normal donors. As a consequence, one unaffected family member (II-6) was identified as heterozygous for the mutation and two affected family members (I-1 and II-3) had the same mutation. None of the 100 population-matched controls had this mutation. The genogram is presented in Fig. 3.

Position and cross-species conservation of T165M. The structure of $F 2$ is reported to be a human prothrombin (5). The affected amino acid (T165M) was identified in exon 6 and resulted in a threonine to methionine substitution at amino acid 165. Previous research has revealed that exon 6 and its flanking regions are highly polymorphic (18). However, T165M was located within a highly conserved region of $F 2$ by means of ClustalW2 which aligned the affected amino acid sequences and the flanking regions (residues 145-185) from human, mouse, orangutan, rabbit, rat, bovine, sheep, horse, pig, tropical-clawed-frog and chimpanzee genomes.

\section{Discussion}

In the present study, CT angiography and clinical trials were used to diagnose thrombophilia within the family. CT 
Table III. The results of a coagulation study in a Han Chinese family with an increased risk of thrombophilia.

\begin{tabular}{|c|c|c|c|c|c|c|c|c|c|}
\hline Subject & $\begin{array}{l}\text { APTT }(\mathrm{sec}) \\
\quad \text { (ref: } \\
28-43.5)\end{array}$ & $\begin{array}{l}\text { PT (sec) } \\
\text { (ref: } \\
11-15.1)\end{array}$ & $\begin{array}{l}\text { PT INR } \\
\text { (ref: } \\
0.75-1.25)\end{array}$ & $\begin{array}{l}\text { PT\% } \\
\text { (ref: } \\
70-120)\end{array}$ & $\begin{array}{l}\text { TT }(\mathrm{sec}) \\
\text { (ref: } \\
14.0-21.0)\end{array}$ & $\begin{array}{l}\text { TT-R ATIO } \\
\text { (ref: } \\
0.67-1 \text { ) }\end{array}$ & $\begin{array}{c}\text { D-DIC }(\mu \mathrm{g} / \mathrm{ml}) \\
(\mathrm{ref}: \\
0-0.5)\end{array}$ & $\begin{array}{l}\text { ATIII\% } \\
\text { (ref: } \\
85-135)\end{array}$ & $\begin{array}{l}\text { Fg }(\mathrm{g} / \mathrm{l}) \\
\text { (ref: } \\
2-4)\end{array}$ \\
\hline $\mathrm{I}-2$ & 43.80 & 13.30 & 1.28 & 69 & 17.80 & 0.85 & 2.85 & 87 & 1.52 \\
\hline II-2 & 44.30 & 12.50 & 1.30 & 68 & 17.40 & 0.83 & 3.94 & 104 & 1.64 \\
\hline II-3 & 43.90 & 14.9 & 1.29 & 77 & 14.10 & 0.75 & 2.78 & 86 & 2.66 \\
\hline II-6 & 32.70 & 12.80 & 1.06 & 105 & 16.30 & 0.69 & 0.11 & 96 & 2.56 \\
\hline II-7 & 20.50 & 11.70 & 1.13 & 112 & 15.40 & 0.71 & 0.06 & 92 & 0.33 \\
\hline III-1 & 44.50 & 13.00 & 1.31 & 70 & 18.30 & 0.82 & 2.55 & 112 & 1.77 \\
\hline III-2 & 31.50 & 12.30 & 1.24 & 107 & 15.20 & 0.75 & 0.01 & 115 & 3.12 \\
\hline IIII-4 & 32.40 & 12.70 & 1.33 & 114 & 14.90 & 0.82 & 0.01 & 121 & 3.34 \\
\hline
\end{tabular}

APTT, activated partial thromboplastin time; ref, reference value; PT, prothrombin time; PT\%, prothrombin time activity percentage; PT INR, prothrombin international normalized ratio; TT, thrombin time; D-DIC, d-dimer; ATIII, antithrombin; Fg, fibrinogen.

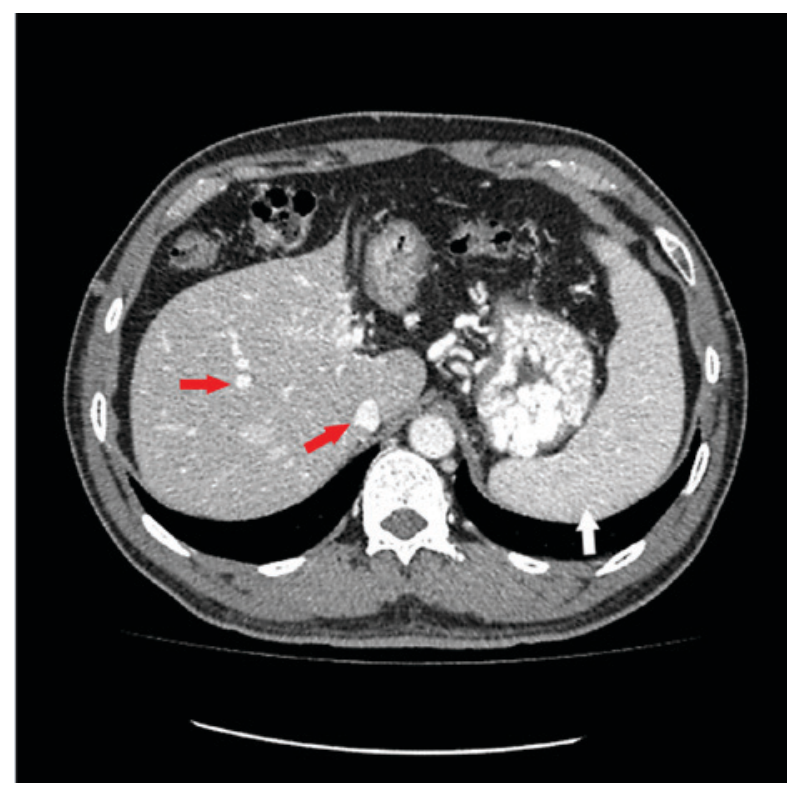

Figure 2. Image from computed tomography angiogram of the proband demonstrating cirrhosis and splenomegaly. (The red arrow indicates liver mass of proband and the white arrow indicates the spleen).

angiography has emerged as a useful diagnostic imaging method for the appraisal of DVT and PE and is more effective than other imaging modalities, particularly for the assessment of mediastinal and parenchymal structures. It was possible to visualize that the location of the inferior vena cava filter and splenomegaly and cirrhosis in the proband. The laboratory measurements may indicate the underlying factor for the greater risk for thrombophilia in this family. Notably, factor V Leiden and prothrombin G20210A mutations were not detected in the proband. The underlying cause may be the resultant abnormalities of blood coagulation components, which may affect the thrombin-mediated mechanism and the process of coagulation and anticoagulation.

The present study investigated the association between the possible $F 2$ variants and the underlying molecular mechanisms of thrombophilia using the WES technique, and

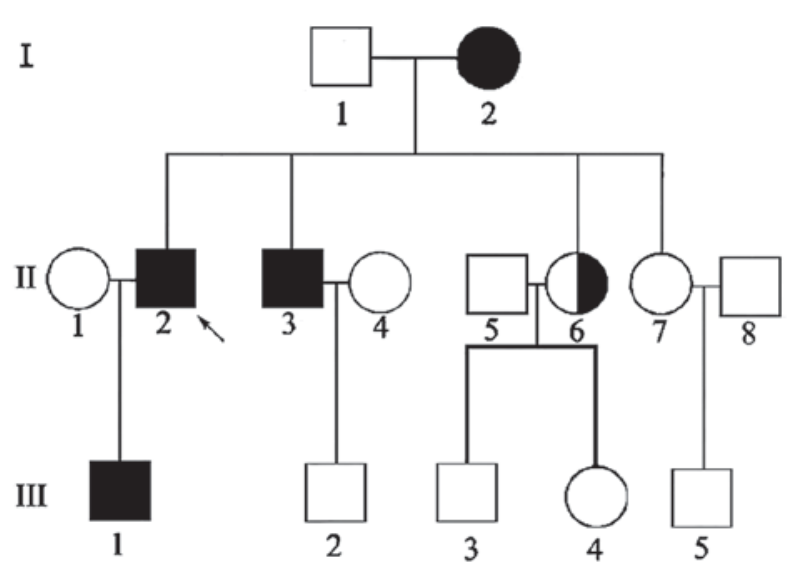

Figure 3. Pedigree of the family with thrombophilia included in the present study. The arrow indicates the proband.

revealed that the $\mathrm{T} 165 \mathrm{M}$ mutation results in a predisposition to thrombophilia. The present study demonstrated that the heterozygous genotype for this variant does not affect the risk of thrombophilia in this Han Chinese family. However, an increased risk of thrombophilia was observed in the homozygotic genotype for this variant. The majority of the thrombophilia-associated gene variants were filtered by a method on the basis of a number of attributes in the present study. Initially, the thrombophilia-associated gene variants were identified in the two affected individuals. Subsequently, the remaining variants were filtered by comparing to the NCBI dbSNP build 132, excluding variants predicted as irrelevant by SIFT and PolyPhen2. This strategy resulted in the identification of the candidate variant in $F 2$. This variant in $F 2$ may result in a form of thrombosis. This site was located in the exon 6 region of $F 2$. The location and missense of the $F 2$ variant suggest that it may exert an effect on the prothrombin function and destroy the vascular integrity during development and postnatal life (from the NCBI Reference Sequence Database). Previous research also observed the T165M variant located in $F 2$ gene in the Xinjiang Kazakhs population, where it was associated with thrombophilia (17). 
In 1996 a mutation in the prothrombin gene, the $F 2$ G20210A mutation, was identified, and this mutation was determined to be a risk factor for thrombophilia (19). In the present study, the T165M variant was observed in the $F 2$ gene, which is the first result to demonstrate this site associated with thrombophilia in $F 2$ gene in the Han Chinese family. The T165M variant was observed in the Han Chinese family studied, and its presence in other affected members besides the proband indicated that this variant may be a risk factor for thrombophilia, and thus thrombosis. In addition, the T165M variant was suggested to be associated with an adverse effect on the prothrombin function and an increased risk of thrombophilia in this Han Chinese family, and has not been identified in previous studies.

The results of the present study may also have other important implications. The T165M variant in the thrombophilia-associated gene $F 2$ was identified as a genetic risk factor for thrombophilia in a Han Chinese family. Therefore, sequencing of the $F 2$ gene using the WES technique may be useful to identify novel genetic defects for thrombophilia, and the present study demonstrates the power of WES as a high-throughput platform for the screening and identification of variants in a time and cost efficient manner. Cross-species amino acid comparison also revealed that the T165M mutation was located in a highly conserved region of $F 2$. As the T165M site was conserved over vast evolutionary distances and this site has been maintained by evolution despite speciation, it is therefore likely to be involved in the normal function of $F 2$.

In conclusion, the results of the present study assisted in the elucidation of the genetic background of thrombophilia. The $F 2$ variant, T165M, was associated with impaired prothrombin function and a genetic risk of thrombophilia in a Han Chinese family. Further studies involving large sample groups remain to be conducted regarding this variant and any possible correlations it may exhibit.

\section{Acknowledgements}

The present study was supported by the Guangxi Natural Science Foundation (grant no. 2015GXNSFBA139176).

\section{References}

1. Raffini L and Thornburg C: Testing children for inherited thrombophilia: More questions than answers. Br J Haematol 147: 277-288, 2009

2. Kvasnička T, Hájková J, Bobčíková P, Cverhová V, Malíková I, Ulrych J, Bříza J, Dušková D, Poletínová S, Kieferová V and Kvasnička J: The frequencies of six important thrombophilic mutations in a population of the Czech Republic. Physiol Res 63 : 245-253, 2014.
3. Coriu L, Ungureanu R, Talmaci R, Uscatescu V, Cirstoiu M, Coriu D and Copaciu E: Hereditary Thrombophilia and thrombotic events in pregnancy: Single-center experience. J Med Life 7: 567-571, 2014.

4. Spiezia L, Campello E, Bon M, Tison T, Milan M, Simioni P and Prandoni P: ABO blood groups and the risk of venous thrombosis in patients with inherited thrombophilia. Blood Transfus 11: 250-253, 2013.

5. Royle NJ, Irwin DM, Koschinsky ML, MacGillivray RT and Hamerton JL: Human genes encoding prothrombin and ceruloplasmin map to 11p11-q12 and 3q21-24, respectively. Somat Cell Mol Genet 13: 285-292, 1987.

6. Walldorf U, Hovemann B and Bautz EK: F1 and F2: Two similar genes regulated differently during development of Drosophila melanogaster. Proc Natl Acad Sci USA 82: 5795-5799, 1985.

7. Al-Allawi NA, Badi AI, Goran MA, Nerweyi FF, Ballo HM and Al-Mzury NT: The contributions of thrombophilic mutations to genetic susceptibility to deep venous thrombosis in iraqi patients. Genet Test Mol Biomarkers 19: 500-504, 2015.

8. Simsek E, Yesilyurt A, Pinarli F, Eyerci N and Ulus AT: Combined genetic mutations have remarkable effect on deep venous thrombosis and/or pulmonary embolism occurence. Gene 536: 171-176, 2014.

9. Cosmi B, Legnani C, Pengo V, Ghirarduzzi A, Testa S, Poli D, Prisco D, Tripodi A and Palareti G; PROLONG Investigators (on behalf of FCSA, Italian Federation of Anticoagulation Clinics): The influence of factor V Leiden and G20210A prothrombin mutation on the presence of residual vein obstruction after idiopathic deep-vein thrombosis of the lower limbs. Thromb Haemost 109: 510-516, 2013.

10. Christiansen SC, Cannegieter SC, Koster T, Vandenbroucke JP and Rosendaal FR: Thrombophilia, clinical factors, and recurrent venous thrombotic events. JAMA 293: 2352-2361, 2005.

11. Zhang Y, Dai Y, Liu Y and Ren J: Mandibulofacial dysostosis, microtia, and limb anomalies in a newborn: A new form of acrofacial dysostosis syndrome? Clin Genet 78: 570-574, 2010.

12. Chen R, Im H and Snyder M: Whole-exome enrichment with the roche nimbleGen SeqCap EZ exome library SR platform. Cold Spring Harb Protoc 2015: 634-641, 2015.

13. Livak KJ and Schmittgen TD: Analysis of relative gene expression data using real-time quantitative PCR and the 2(-Delta Delta C(T)) Method. Methods 25: 402-408, 2001.

14. Clark MJ, Chen R, Lam HY, Karczewski KJ, Chen R, Euskirchen G, Butte AJ and Snyder M: Performance comparison of exome DNA sequencing technologies. Nat Biotechnol 29: 908-914, 2011.

15. Ng PC and Henikoff S: SIFT: Predicting amino acid changes that affect protein function. Nucleic Acids Res 31: 3812-3814, 2003.

16. Adzhubei I, Jordan DM and Sunyaev SR: Predicting functional effect of human missense mutations using polyPhen-2. Curr Protoc Hum Genet Chapter 7: Unit7.20, 2013.

17. Ge XH, Zhu F, Wang BL, Wang CM, Zhu B, Guan S, Ci HB, Sai LM, Jiang XK and Ren H: Association between prothrombin gene polymorphisms and hereditary thrombophilia in Xinjiang Kazakhs population. Blood Coagul Fibrinolysis 25: 114-118, 2014.

18. Iwahana $\mathrm{H}$, Yoshimoto $\mathrm{K}$ and Itakura M: Highly polymorphic region of the human prothrombin (F2) gene. Hum Genet 89: 123-124, 1992.

19. Poort SR, Rosendaal FR, Reitsma PH and Bertina RM: A common genetic variation in the 3'-untranslated region of the prothrombin gene is associated with elevated plasma prothrombin levels and an increase in venous thrombosis. Blood 88: 3698-3703, 1996. 